this way. The ability of parents and friends to perceive distress in adolescents appears to vary with social class.

At a time when there has been much revised legislation dealing with the management of young offenders it is surprising that only six teenagers were referred by the court for psychiatric assessment. The setting up of family courts as recommended In the Kilbrandon Report (1964) and the establishment in Edinburgh of a unit specifically for adolescent psychiatric illness should improve the situation.

\section{Summary}

A survey is reported of referrals of adolescents to psychiatrists in Edinburgh. The annual referral rate was 5.6 per thousand at risk. For one-third of the series the consultation took place after an act of deliberate self-poisoning or self-injury ; almost one-half were referred by their family doctor and one-tenth were self-referrals. The social and emotional difficulties arising from the natural hesitance of adolescents to discuss their problems with adults are discussed.
We are grateful to Professor G. M. Carstairs for his help and advice ; to Drs. Norman Kreitman and John Evans for their criticism and advice; and to the physician-superintendents and consultant psychiatrists in the region.

\section{REFERENCES}

Backett, E. M., Shaw, L. A., and Evans, J. C. G. (1953). Proc. roy. Soc. Med., 46, 707.

Hare, E. H., and Shaw, G. K. (1965). Mental Health on a New Housing Etate. London.

Kessel, W. I. N. (1960). Brit. F. prev. soc. Med., 14, 16

Kilbrandon Committee (1964). Children and Young Persons, Scotland. Scottish Home and Health Department, Scottish Education Department. H.M.S.O., London.

Philip, A. E., and McCulloch, J. W. (1966). Brit. F. prev. soc. Med., 20, 122.

Siegel, S. (1956). Nonparametric Statistics for the Behavioural Sciences. New York.

Srole, L., Langner, T. S., Michael, S. T., Opler, M. K., and Rennie, T. A. C. (1962). Mental Health in the Metropolis: The Mid-town Manhattan Study, vol. 1. New York.

Taylor, Lord, and Chave, S. (1964). Mental Health and Environment. London.

Toolan, J. M. (1962). Amer. F. Psychia:., 118, 71.9.

Toolan, J. M. (1962). Amer.
Warren, W. (1965). F. Child Psychol., 6, 1.

\title{
Standardization of the One-stage Prothrombin Time for the Control of Anticoagulant Therapy
}

\author{
ROSEMARY BIGGS,* M.D. ; K. W. E. DENSON,* D.PHIL.
}

Brit. med. 9., 1967, 1, 84-88

The one-stage prothrombin time test is widely used for the control of anticoagulant therapy and has proved on the whole satisfactory for this purpose. One difficulty is that the results are expressed in many different ways, and even if this is done apparently in the same way the results of one centre are seldom directly comparable to those of another. Thus if a patient moves from one centre to another there may be a change in the degree of anticoagulation. Another difficulty is that clinical trials carried out at more than one hospital may be difficult unless special steps are taken to standardize the method.

In recent years efforts have been made to solve this problem, and co-ordination should greatly improve the situation. In the United States the College of American Pathologists has reviewed the techniques in use and made some recommendations about acceptable techniques (Miale and Lafond, 1963). The Committee for Haemostasis and Thrombosis is devising a method of comparing results based on a standard thromboplastin (Biggs, 1965 ; Biggs and Denson, 1966). In England Poller (1964) has been studying the preparation of a reagent to be used in a wide area, so that all the hospitals shall have a unified method. These studies have been made from rather different points of view and are complementary to each other rather than competing, and consequently a much more constructive approach should be possible.

\section{Coagulation Defect caused by Coumarin Drugs}

The coumarin drugs cause variable reduction in the blood concentrations of clotting factors VII, IX, X, and prothrombin (II). The one-stage prothrombin time records abnormality in factors II, VII, and X, and thus in most patients gives a fair

- Medical Research Council, Blood Coagulation Research Unit, Churchill Hospital, Oxford. record of the abnormality. Since the defect is composite and it is impracticable to measure all of the factors separately, an overall assessment of the abnormality is made from the results of the test.

\section{Possibility of Standardization}

The details of technique will not be discussed, but in all of the methods the abnormality is recorded as a lengthening of clotting-time of the patient's plasma in comparison with normal. Table I gives examples of results obtained with different methods. One difficulty in comparing one method with another is clearly the different clotting-times of normal plasma -the results in Table I show a range from 13.2 to 45 seconds. This difficulty is avoided if the clotting-time ratios are calculated (Table I), because all of the normal clotting-times are expressed as a clotting-time ratio of 1 . It will be seen from Table I that if the ratios are taken as an expression of the degree of abnormality, then the methods vary a good deal in this

TABLE I.-One-stage Prothrombin Time was Carried Out on a Normal Plasma Sample and on Three Samples from Patients Receiving Anticoagulant Therapy. Six Different One-stage Techniques wero Used. The Clotting-times Obtained are Expressed in Seconds; the Clotting-time ratio is calculated by Dividing the Clotting-time for the Abnormal Sample by that of the Normal (e.g., $\frac{56}{45}=1.25$ )

\begin{tabular}{|c|c|c|c|c|c|c|c|c|c|c|c|c|}
\hline \multirow{2}{*}{ Sample } & \multicolumn{2}{|c|}{ Method 1} & \multicolumn{2}{|c|}{ Method 2} & \multicolumn{2}{|c|}{ Method 3} & \multicolumn{2}{|c|}{ Method 4} & \multicolumn{2}{|c|}{ Method 5} & \multicolumn{2}{|c|}{ Method 6} \\
\hline & C. & R. & C. & $\mathrm{R}$. & C. & R. & C. & R. & c. & R. & C. & R. \\
\hline Normal & 45 & $1 \cdot 0$ & 44 & $1 \cdot 0$ & $13 \cdot 2$ & $1 \cdot 0$ & 19 & $1 \cdot 0$ & $19 \cdot 7$ & $1 \cdot 0$ & 14 & $1 \cdot 0$ \\
\hline $\begin{array}{l}1 \\
\text { Patient }\end{array}$ & 56 & $1 \cdot 25$ & 50 & $1 \cdot 13$ & 14 & 1.08 & 21 & $1 \cdot 1$ & $22 \cdot 5$ & $1 \cdot 24$ & $15 \cdot 7$ & $1 \cdot 12$ \\
\hline $\begin{array}{c}2 . . \\
\text { Patient }\end{array}$ & 120 & $2 \cdot 65$ & 96 & $2 \cdot 2$ & 19 & $1 \cdot 44$ & 35 & $1 \cdot 85$ & 38 & 1.94 & 21.5 & 1.54 \\
\hline $3 \ldots$ & 106 & $2 \cdot 35$ & 82 & 1.87 & $18 \cdot 8$ & $1 \cdot 42$ & 38 & $2 \cdot 0$ & 33 & 1.68 & 21 & 1.72 \\
\hline
\end{tabular}


respect. This is a phenomenon well known to coagulation workers and is one of the main reasons why it is difficult to compare one method with another.

If comparative tests are carried out on a large number of samples from patients on anticoagulant therapy by different methods a certain regularity is disclosed. If the clotting-time ratios obtained by two methods are plotted one against the other a straight line can be drawn through the points (Fig. 1). From the results shown in Fig. 1 it would be reasonable to say that on average a clotting-time ratio of 2 by method 1 was equivalent to one of 2.2 by the second method and that a ratio of 3 was equivalent to one of 3.45 , and so on. This process of comparison has been made on a very large number of samples (Biggs, 1965; Biggs and Denson, 1966) and the relation has been found to hold good by various methods and in many different laboratories. This regularity observed in comparing techniques means that it should be possible to calibrate all methods in terms of a standard. The problem is to establish and define the standard.

\section{Expression of Results}

Most of the commercial methods provide a calibration curve which converts the results to " percentage of normality." These dilution curves do not ensure any uniformity between methods of assessing the degree of abnormality, because the sensitivity of the methods to the coumarin defect is not truly represented by these dilution curves. The curves are made by testing the clotting-times by the one-stage technique of dilutions of normal plasma, and they vary very considerably according to the diluent used, to the nature of the thromboplastin, and to other details of technique. To many the dilution curve has seemed such an arbitrary scale of measurement that the use of simple clotting-time ratios have been preferred. Thus it may be the objective to maintain a therapeutic range of two to two and a half times the normal clotting-time.

The ratio method has the advantage that it does not carry any unjustified implication of scientific validity. But the dilution curve and ratio methods of calculating the results are unlikely to give the same information. This can be visualized by reference to Fig. 2, which presents hypothetical dilution curves for two modifications of technique. If the clinician

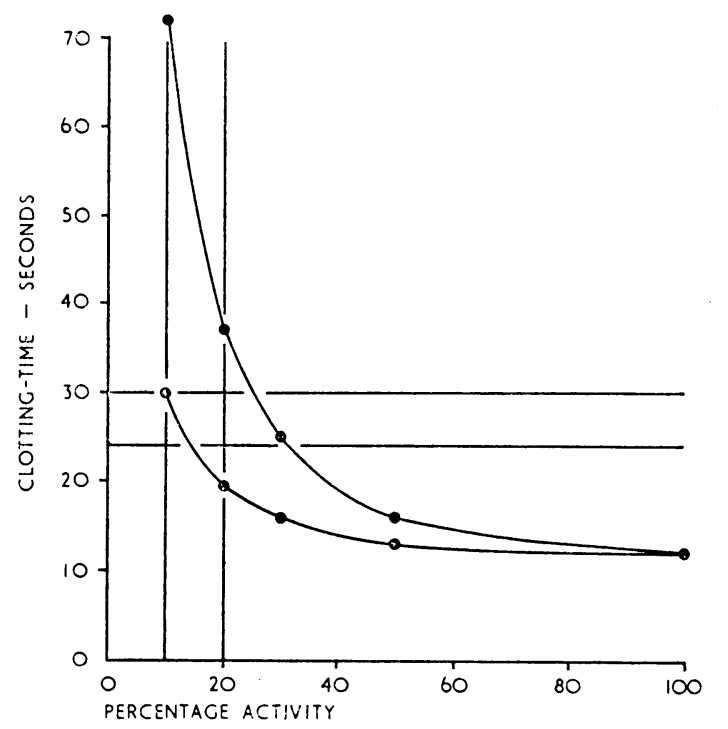

FIG. 2.-Two hypothetical dilution curves for the one-stage prothrombin-time method. The horizontal lines show the limits of the "therapeutic range" if the criterion used is limits of the "therapeutic range if the criterion used is tino to two and a half times the clotting-time. The vertical lines show the range if the criterion is 10 to $20 \%$ of activity Unless the dilution curves for the methods are identical the two criteria must give a different degree of abnormality.

decides to maintain a therapeutic range of two to two and a half times the normal clotting-time this will represent quite different activities if the dilution curves are taken to represent the true abnormality. The difference is delineated by the horizontal lines in Fig. 2, which includes the range two to two and a half times the clotting-time. If, on the other hand, the clinician decides to use a percentage range, say 10 to $20 \%$ of normality, then clearly the two methods will differ in the ratios equivalent to this range.

These dilemmas about the expression of the results of the one-stage prothrombin time are well known, and it is towards a satisfactory solution to them that the efforts of standardization are directed. The aim should be to make a realistic assessment of the dilution curve for a particular method, because if the clotting-time means anything (as it certainly does) then it is the amount of residual activity which is important.

The dilution curve is thus the central point in this study. In 1949 Biggs and Macfarlane pointed out that the dilution curve could be expressed linearly by plotting the clottingtime against the reciprocal of the concentration of whole plasma in the diluted mixtures. More recently it has also been shown (Biggs, 1965) that the clotting-time ratios for the dilution curve can be expressed linearly. This point is illustrated by making this mathematical conversion for a number of dilution curves (Fig. 3). This method of expressing the results has the advantage that the normal is expressed as unity by all methods. This mathematical conversion does not of course solve the problem of which dilution curve is appropriate to a particular method for the control of anticoagulant therapy.
FIG. 1.-One-stage prothrombin-time investigation was carried out on 20 samples from patients receiving anticoagulant therapy with six different test methods and a standard method. The clotting-time ratios were calculated, and for each of the methods the ratio was plotted against give the best average results in comparing the two methods. 
It is, however, possible to put together the results of correlation studies, using different methods, with this linear method of expressing the dilution curve. Let us take, for example, the results in Fig. 1, which shows correlation graphs of six different mothods and a standard method. From these graphs an average equivalence between the methods can be worked out. For methods 1 and 3 of Fig. 1 these equivalent ratios are given in Table II. If a dilution curve is drawn for the standard method, then, on the basis of the figures in Table II, dilution curves

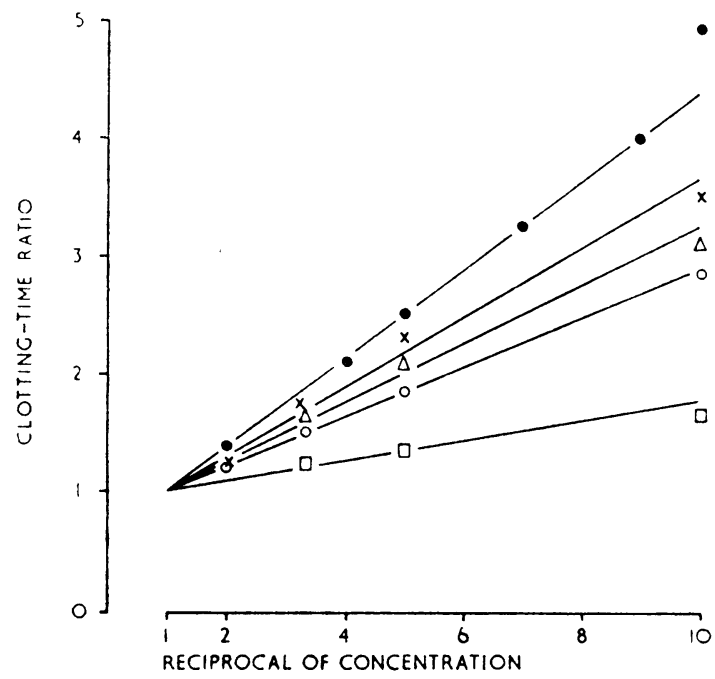

Fig. 3.-Dilution curves for a variety of methods are presented by plotting the reciprocal of normal plasma concentration against the clotting-time ratio. The lowest curve was obtained with human brain extract by diluting normal plasma with plasma from a patient with very severe vitamin-K deficiency. The other curves are derived from curves issued with commercial scale reagents. It should curves issued with cominercial scale reagents. It should the sensitivity of the methods to the defect caused by coumarin drugs.

can be drawn for methods 1 and 3 (Fig. 4). It will be seen that if the standard method is expressed as a straight line then use of the correlation points from Fig. 1 produces straight-line graphs for methods 1 and 3 .

If the dilution curve appropriate to each method is used the clotting-times for the individual samples used for Fig. 1 may be converted to percentage of normality. The results of this

TABLB II.-Ratios Equivalent to the "Standard" Method of Fig. 1 are Taken from the Solid Circles of Fig. 1

\begin{tabular}{c|c|c}
\hline Ratio by Standard Method & Ratio by Method 1 & Ratio by Method 3 \\
\hline 2 & $2 \cdot 2$ & 1.4 \\
3 & 3.45 & 1.75 \\
4 & 4.65 & 2.1 \\
5 & 5.85 & 2.45 \\
6 & 7.1 & 2.85
\end{tabular}

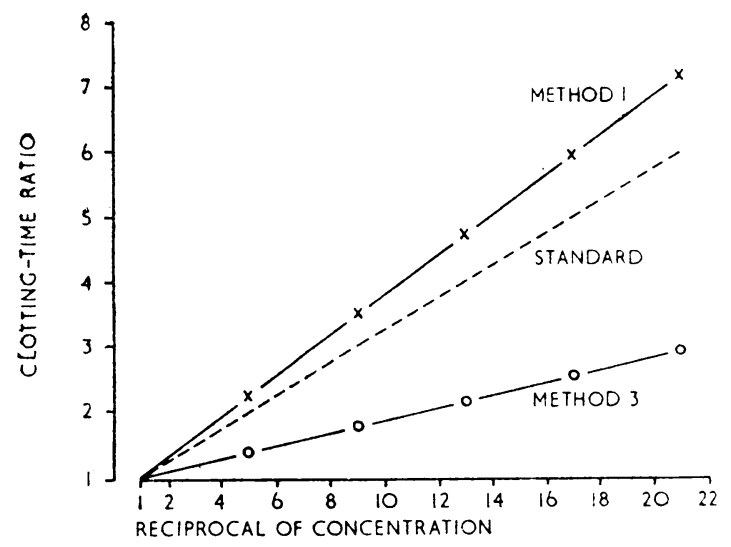

Fig. 4-Calibration graphs for the standard method and methods 1 and 3 from Fig. 1 conversion are given in Table III. The correspondence between the results of the methods using appropriate dilution curves is obvious. This particular example includes a number of very long clotting-times and was chosen for this reason to illustrate the correlation graphs ; thus many of the "percentage" results are low. This sort of correlation has in fact been made with very many samples over a wide range of clotting-times and seems to hold generally (Biggs, 1965).

TABLE III.-Percentage Results for Two Different One-stage Methods Compared with Those of an Arbitrarily Selected Standard Method Using the Dilution Curves of Fig. 4 to Ascertain the Percentages

\begin{tabular}{c|c|c|c} 
Sample & Standard & Method 1 & Method 3 \\
\hline 1 & 20 & 23 & 18 \\
2 & 16 & 16.5 & 15 \\
3 & 12 & 12.5 & 12 \\
4 & 11 & 11 & 11.5 \\
5 & 10.5 & 11 & 13 \\
6 & 10.5 & 9.5 & 11 \\
7 & 9 & 9 & 11 \\
8 & 9 & 9 & 11 \\
9 & 9 & 8.5 & 9 \\
10 & 8.5 & 8 & 9.5 \\
11 & 8.5 & 7.5 & 7.5 \\
12 & 7.5 & 6 & 6.5 \\
13 & 6 & 6 & 6.5 \\
14 & 6 & 5 & 7.5 \\
15 & 5 & 5 & 7.5 \\
16 & 5 & $<5$ & 6.5 \\
17 & 5 & $<5$ & $<5$ \\
18 & $<5$ & $<5$ & $<5$ \\
19 & $<5$ & & \\
20 & $<$ & & 5 \\
& & 5 &
\end{tabular}

Since the standard is to be used merely as a basis for unified comparison, this method of rendering the results of one technique on a scale adjustable to all can form the basis of the standardizing procedure ; it remains to select a standard. Fig. 4 shows that the methods differ from each other in the steepness of the line expressing the dilution curve. It is easy to see that the steeper the line the more "sensitive" the method to the defect caused by the coumarin drugs. The reductio ad absurdum would be a line along the base showing no alteration in clotting-time with treatment. Thus a satisfactory method to use as a standard is one with a good sensitivity and a steep line. For general purposes a method with a reasonably short clotting-time for normal plasma would probably be most generally acceptable.

\section{Standard Preparation}

A standard preparation based on a good deal of preliminary work can now be proposed. This is a reagent containing absorbed ox plasma and using human brain as a source of thromboplastin. This method is provided with an arbitrary dilution curve relating clotting-time ratios with percentage of normality, which has been found in practice to cover the most usually employed degrees of anticoagulation. Such a curve based on ratios is shown in Fig. 5. This can probably more usefully be presented as a Table (Table IV).

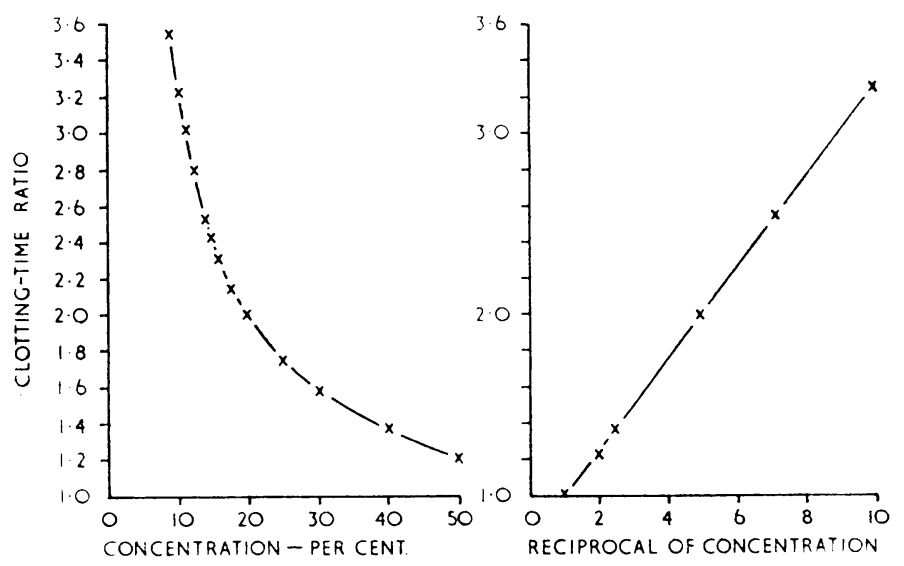

Fig. 5.-Dilution curve for the standard method. 
TABLE IV.-Clottıng Time Ratios Equivalent to Various Arbitrary Percentages for the Standard Method

\begin{tabular}{c|c|c|c|c|c}
\hline $\begin{array}{c}\text { Clotting- } \\
\text { time Ratio }\end{array}$ & $\%$ & $\begin{array}{c}\text { Cloting- } \\
\text { time Ratio }\end{array}$ & $\%$ & $\begin{array}{c}\text { Clotting- } \\
\text { time Ratio }\end{array}$ & $\%$ \\
\hline 1.0 & 100 & 2.14 & 18 & 3.02 & 11 \\
1.25 & 50 & 2.23 & 17 & 3.25 & 10 \\
1.37 & 40 & 2.32 & 16 & 3.54 & 9 \\
1.59 & 30 & 2.44 & 15 & 3.88 & 8 \\
1.76 & 25 & 2.54 & 14 & 4.34 & 7 \\
2.0 & 20 & 2.67 & 13 & 4.93 & 6 \\
2.08 & 19 & 2.82 & 12 & 5.72 & 5 \\
\hline
\end{tabular}

\section{Use of the Standard Preparation in Practice}

The standard preparation should be used in parallel with the method preferred in a particular laboratory on a number of normal samples and samples from patients treated with the coumarin anticoagulant in use. An example of such a comparative study is presented in Table $\mathrm{V}$. The figures for the

TABle V.-Twenty-one Plasma Samples from Patients Receiving Anticoagulant Therapy were Tested by Two Methods, One of Which was Taken as the Standard. The Clotting-time Ratios and Percentages from Appropriate Calibration Tables are Given. These Figures are Presented in Figs. 6 and 7

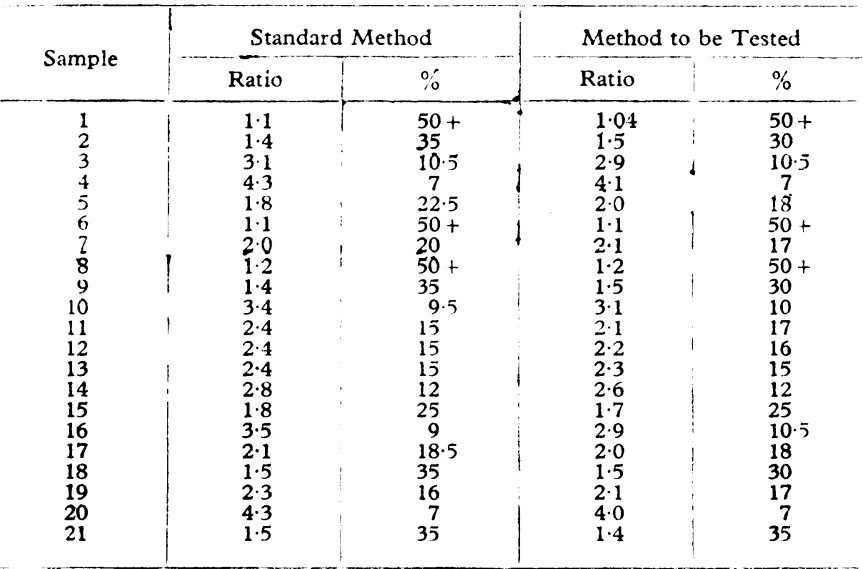

two methods are plotted against each other and the best line to fit the points is drawn (Fig. 6). The ratios of the two methods are then compared. If the ratio of 2 by the standard method is taken as a fixed point, then by the second method it can be seen that a ratio of 1.9 would indicate the same degree of abnormality. The appropriate dilution curve for the method can thus be fixed, or perhaps more conveniently a table of ratio equivalents to percentage may be used. Table VI presents a composite set of figures which could be used for all methods. At the top of this table are given the figures for the ratio equivalent to that of the standard ratio of 2 . In the example given this was 1.9. Thus for this method the column headed 1.9 would be used. The figures for conversion to percentages appear in Table V. In Fig. 7 the percentage results for the two methods have been plotted; clearly, if the therapeutic range is taken to be 10 to $20 \%$ both methods give a satisfactory assessment of the samples.

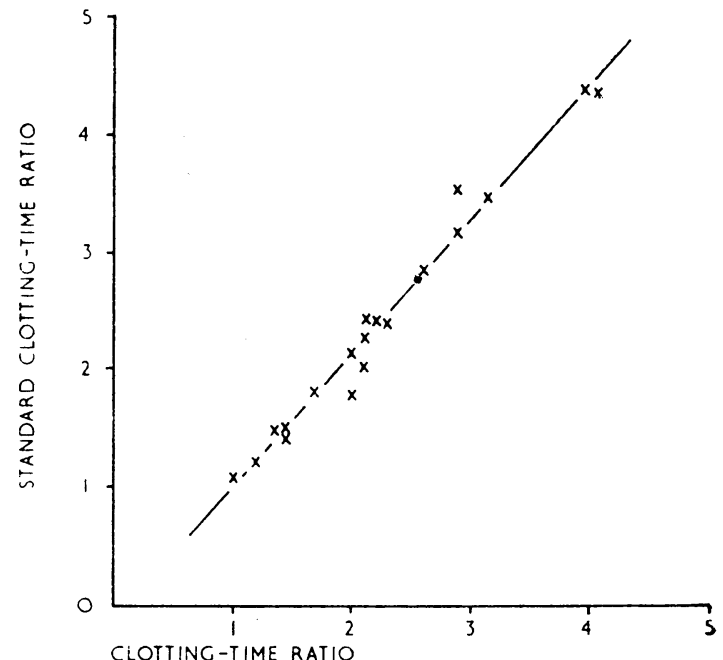

FIG. 6.-The one-stage method was carried out on a number of samples from patients having anticoagulant therapy, a "standard" method and a test method being used, and the clotting-time ratios for the two methods are plotted against each other.

\section{Commercial Scale Preparations}

The principle of the method of standardization depends quite simply on the comparison of the method in question with a reference preparation of known sensitivity. The two methods are used on a number of samples from patients receiving anticoagulant therapy and the results compared as in Table $\mathrm{V}$ and Fig. 6. The method could very well be used to standardize commercial thromboplastins, which would then be issued with an approved scale.

\section{Minimum Standard of Sensitivity}

A preparation and method which is insensitive to the defect caused by coumarin drugs can never give satisfactory results. At present the majority of preparations are calibrated more by their reaction to saline dilutions of normal plasma than by their sensitivity to the defect caused by coumarin drugs. Thus some

TABLE VI.-Calibration Table for Use with the One-stage Prothrombin-time Methods of Varying Sensitivity to the Coumarin Defect

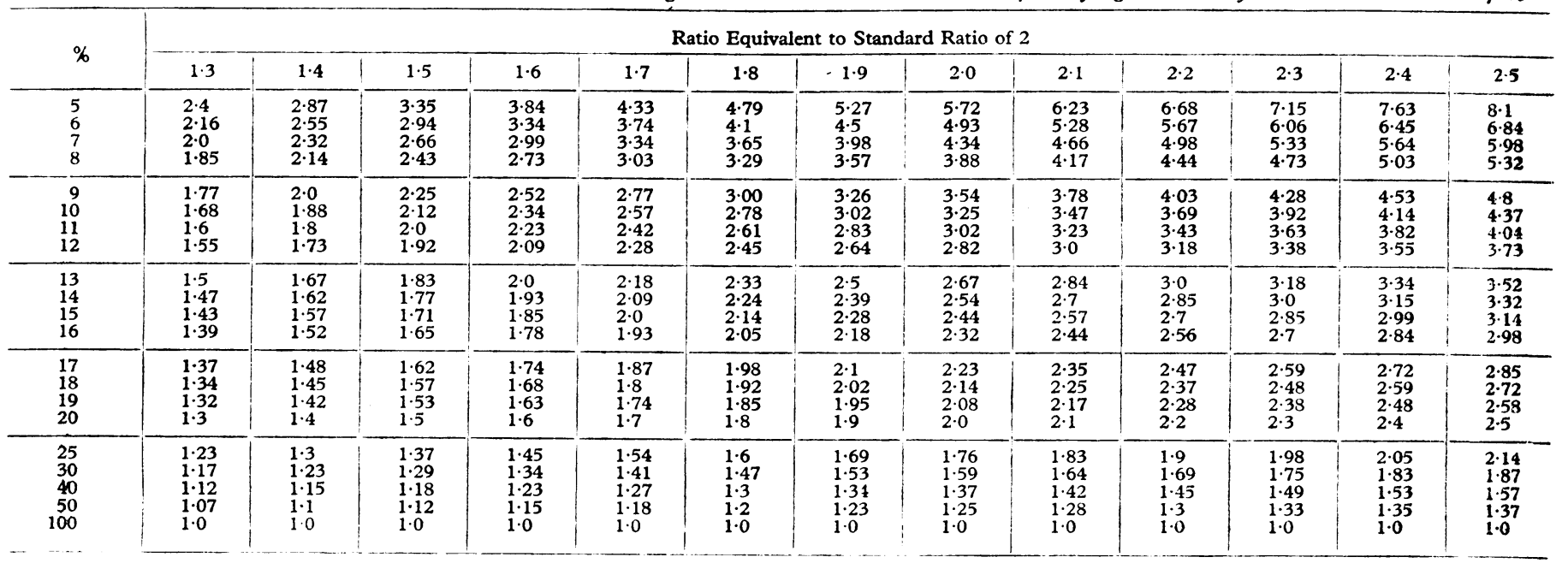


commercial preparations are very insensitive. When the first reference preparation is available it would be an advance for a minimum acceptable sensitivity to be established by standards institutions. Such a concept would encourage the preparation of satisfactory commercial preparations.

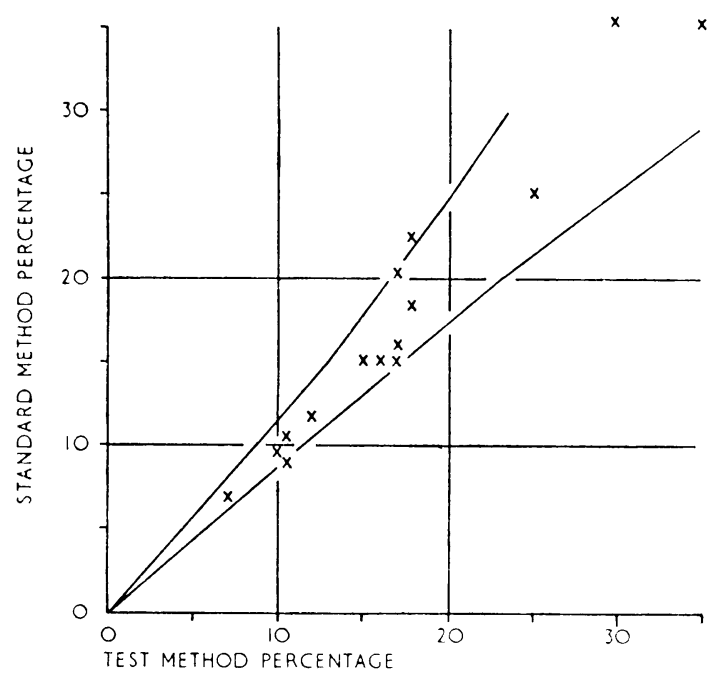

FIG. 7.- Results presented in Fig. 6 converted to percentage activity from appropriate calibration scales and the results plotted against each other. The vertical and hori zontal lines give a reasonable "therapeutic range." The limiting lines show the range of error to be expected from the method as a result of simple inaccuracy in determining the clotting-time.

\section{Conclusion}

The work done in many centres on the standardization of the one-stage prothrombin time for the control of anticoagulant therapy with coumarin drugs is reaching a stage when it may be possible greatly to improve the general standard of control.

The emphasis on technical care in carrying out the test, always noted by Quick and more recently underlined by Miale, is clearly important. The use of the same technique in a wide area also obviously has great advantages (Poller, 1964). The introduction of a reference preparation with an internationally recognized scale and a simple method of adjusting various techniques to the scale would round off this work. The calibration scale of commercial preparations could be adjusted to the scale, preparations made individually in clinical laboratories could be similarly calibrated. Moreover, attention to sensitivity to the coumarin defect would undoubtedly improve the reliability of control.

A single scale properly applied at different centres would ensure safety and uniform dosage for a patient moving from one place to another and would greatly improve the standard of clinical trials carried out at more than one centre.

\section{Summary}

The control of anticoagulant therapy by the one-stage prothrombin time suffers from the fact that the results of this test are reported differently at different centres. Thus clinical trials with a standard objective of treatment are difficult to organize and a patient cannot travel from one centre to another with any assurance that the level of therapy will remain constant. A method of standardizing the test has been devised so that all may use the same scale of measurement adjusted in each case to fit the preferred thromboplastin. The method is based on the preparation of the standard thromboplastin with which other preprarations can be compared.

The work reported in this paper was undertaken jointiy on behalf of the M.R.C. Working Party on Anticoagulant Therapy and the International Committee on Haemostasis and Thrombosis.

\section{REFERENCES}

Biggs, R. (1965). Transactions of the Conference Held under the Auspices of the International Committee on Blood Clotting Factors, 1964, pp 303-327.

and Denson, K. W. E. (1966). Transactions of the Conference Held under the Auspices of the International Committee on Haemostasis and Thrombosis, 1965.

- and Macfarlane, R. G. (1949). 7. clin. Path., 2, 33

Poller, L. (1964). Brit. med. F., 2, 565.

Miale, J. B., and Lafond, D. J.' (1963). Prothrombin Survey, College of American Pathologists. Standards Committee: Subcommittee on Coagulation.
According to the W.H.O. definition, dextromoramide would fall into the class of drugs producing dependence of the morphine type. The most characteristic feature of this type of dependence is production of the abstinence syndrome within a few hours of administration of the last dose of the drug ; the symptoms which occur in this syndrome are described in the W.H.O. report and by Eddy, Halbach, Isbell, and Seevers (1965). They can be evaluated by the Himmelsbach (1939) scale. The W.H.O. report states that the abstinence syndrome may also be precipitated in a matter of minutes by the administration of a specific antagonist while continuing to administer the agent responsible for the dependence. Wikler, Fraser, and Isbell (1953) have shown that the administration of nalorphine can act as a specific antagonist to morphine, methadone, and heroin in this way. 